\title{
Tres traducciones españolas de Kader Abdolah
}

\section{Three Spanish translations of Kader Abdolah}

\author{
Philippe HuMBLÉ \\ Vrije Universiteit Brussel \\ Philippe.Humble@vub.ac.be
}

Recibido: 12-2-2016. Revisado: 26-10-2016. Aceptado: 1-11-2016.

Resumen: En este artículo se estudian las traducciones al español de tres novelas de Kader Abdolah, un escritor que tiene la particularidad de ser emigrante y de expresarse en una lengua, el neerlandés, que no es la suya. En lugar de tratar de escribir como el resto de los autores del país donde reside, Kader Abdolah se sirve de su lengua de adopción para recalcar su identidad de extranjero, alcanzando un gran éxito editorial. En el presente trabajo se examina de qué forma el peculiar neerlandés de Abdolah aparece traducido al español en las novelas El viaje de las botellas vacías, La casa de la mezquita y El reflejo de las palabras. En concreto, se analiza la relación type/token, que nos informa sobre la densidad léxica del original comparado con la traducción. Así se descubre que todos los traductores, aun traduciendo de manera diferente, «enriquecen» el texto original, a diferencia de lo que sugiere la hipótesis de los translation universals. El resultado es que la falta de «riqueza léxica», una característica propia del autor, se pierde en la traducción. Será, por lo tanto, el propio relato el que tendrá que garantizar al escritor la transmisión del relato, y no su peculiar estilo.

Palabras clave: Traducción literaria; literatura inmigrante; traducción neerlandés-español; universales de la traducción; densidad léxica. 
Abstract: This study of three novels by Kader Abdolah translated into Spanish - El viaje de las botellas vacías, La casa de la mezquita and El reflejo de las palabras - examines how the author's non-native Dutch is conveyed in a foreign language. Rather than attempting to write like native Dutch author, Abdolah, an immigrant to the Netherlands, has used his adopted language to underline his alien identity and has become a topselling autor. The type/token ratio, a measure that can reveal the lexical density of source texts versus target texts, is analyzed and discussed. The target-text analysis of the three-novel corpus reveals that all translators end up "enriching" the texts in such a way that the low «lexical diversity» of the source texts, a cornerstone of the author's "exile style», is lost in Spanish. This tendency runs counter to what the hypothesis of translation universals predicts. Kader Abdolah will have to rely on the plot of his novels rather than on his singular style to convey his message.

Keywords: Literary translation; migrant literature; Dutch-Spanish translation; translation universals; lexical density.

\section{INTRODUCCIÓN}

Parece evidente que el neerlandés, con 25 millones de hablantes, es una lengua «de menor difusión», una lengua «minoritaria», sobre todo al compararla con una lengua como el español. Parece igualmente natural, por lo tanto, que en los Países Bajos y Bélgica se traduzca más del español al neerlandés que en los países hispanohablantes del neerlandés al español.

Hablar de «lengua minoritaria» implica una valoración y no depende únicamente del número de hablantes. Una lengua es «minoritaria» porque se la compara con otras, «mayoritarias». No es un dato objetivo, ya que uno puede hablar de una lengua minoritaria aun siendo la lengua de millones de personas. El hindi es la lengua materna de casi 300 millones de personas, pero sigue siendo una lengua minoritaria. Hasta el chino, con más de mil millones de hablantes, puede ser considerado una lengua minoritaria, ya que del chino se traduce poco. Una lengua minoritaria puede ser una lengua hablada por pocos, o culturalmente poco valorizada. La clasificación como lengua mayoritaria, o dominante, tiene relación con la importancia económica, política y cultural de los países donde esa lengua se habla, y con el número de personas que la usa como segunda lengua.

Ahora bien, la realidad demográfica es un dato en el que apenas se puede influir porque depende en primera instancia de la fecundidad de las familias (o de las ganas de tener hijos). Por otro lado, es el dinero el que otorga el poder económico, y el político-

militar está relacionado con este, aunque no directamente. No en vano Casanova $(2015,123)$ afirma: «On associe souvent la puissance économique et militaire à la puissance linguistique; or elles sont nettement distinctes, l'une servant seulement à renforcer l'autre».

Philippe HUMBLÉ Tres traducciones españolas de Kader Abdolah 
Es el ser humano el que otorga prestigio cultural. El que una lengua sea mayoritaria desde el punto de vista de la cultura depende del prestigio que se concede a esa lengua. En Europa, el francés fue la lengua con mayor prestigio cultural después del ocaso del latín a partir del siglo XVI, y este prestigio se fundamentaba solo en parte en su poderío económico y militar. El francés siguió dominando como vehículo de comunicación cultural en gran parte del mundo hasta hace poco. El prestigio cultural se cimienta básicamente en la opinión de algunos, de los hombres de letras, de los legisladores culturales que son los críticos, los periodistas, los académicos, los escritores. Esta "opinión", difícil de definir, se basa en hechos que son en parte culturales, pero que también se derivan del respeto que infunden a todos las «cualidades» económicas y militares de una nación. ¿Por qué, si no, fue España un país exportador de cultura en el Siglo de Oro para después convertirse en importador?

Por ahora vivimos en una era de supremacía cultural norteamericana que no justifica ni la calidad de su producción literaria, musical, plástica, ni tampoco sus derivados: la música popular, las películas, las series televisivas, la publicidad. Aparentemente, se trata de una elección dictada por razones ajenas a la propia cultura. Si todas las culturas son igual de válidas y si, a pesar de eso, una de ellas prevalece sobre las otras en un momento determinado de la historia, no es por razones intrínsecamente culturales. Ya lo decía Borges (1974, 773): «Mi desconocimiento de las letras malayas o húngaras es total, pero estoy seguro de que si el tiempo me deparara la ocasión de su estudio, encontraría en ellas todos los alimentos que requiere el espíritu». Dicho de otro modo, no hay criterios objetivos que permitan afirmar que una cultura sea superior a otra.

Ciertas lenguas gozan de más prestigio que otras, por razones no siempre del todo claras. China es una potencia económica desde hace algunas décadas. Sin embargo, no asistimos a una avalancha de traducciones literarias del chino, y posiblemente esto no se deba en exclusiva a que haya pocos traductores que trabajen con esa lengua. Del mismo modo, si bien es cierto que los estudiosos chinos están cada vez más presentes, por ejemplo en el área de la teoría de la traducción, cabe puntualizar que no escriben en chino, sino en inglés.

¿Y el neerlandés? Es una de esas lenguas a las que los editores no suelen prestar demasiada atención. En muchos casos, el interés viene motivado por las subvenciones concedidas por la fundación dedicada a la promoción de la literatura de los Países Bajos en el mundo (Nederlands Letterenfonds) o de su homóloga flamenca (Vlaams Fonds voor de Letteren).

Del inglés, por otro lado, sabemos que se traduce mucho. ¿Y al inglés? Los datos relativos a los textos literarios más traducidos en el Reino Unido nos dan una buena idea de la popularidad de las otras lenguas en el mundo:

Philippe HUMBLÉ Tres traducciones españolas de Kader Abdolah 
[...] source language analysis of the processed data shows that the top ten translated European languages are French, German, Spanish, Russian, Italian, Swedish, Norwegian, Dutch, Portuguese and Danish. The gap between the leading language, French, and the second one, German, is around 40\%. The top five non-European languages are Arabic, Japanese, Chinese, Hebrew and Persian. Measured globally, the first two make it into the top ten, pushing the last two European languages out of the «top ten league». (Büchler y Trentacosti 2015, 5)

Si la diferencia entre el francés y el alemán es de un 40\%, se espera que el número de obras literarias traducidas del neerlandés sea aún mucho menor, pese a que la lengua neerlandesa figura entre las diez lenguas europeas más traducidas al inglés. De acuerdo con la información recogida en la base de datos del Nederlands Letterenfonds y el Vlaams Fonds voor de Letteren ${ }^{1}$, entre 2000 y 2015 se tradujeron del neerlandés al inglés 386 obras de ficción, una cantidad muy superior a las 158 obras traducidas del neerlandés al español y publicadas en España o Hispanoamérica en ese mismo período.

Stella Linn (2006, 27-28) afirma en un artículo dedicado a las relaciones literarias entre el neerlandés y las demás lenguas, desde el punto de vista de la traducción: «In recent years the number of works translated into Dutch has been almost six times those translated from Dutch». La situación no debe de haber cambiado mucho en los diez años transcurridos desde que Linn publicara su artículo. Las lenguas «minoritarias» siguen ocupando un espacio reducido en los fondos publicados por las editoriales de las lenguas «mayoritarias». Aun así, algunos consiguen romper barreras. De hecho, autores como Cees Nooteboom, Arnon Grunberg o Herman Koch tienen éxito también en español, pero son los menos.

\section{KADER ABDOLAH}

A la luz de lo anterior, resulta sorprendente que Kader Abdolah, un escritor holandés-iraní, haya conseguido publicar tres libros en español. Resulta tanto más sorprendente si tenemos en cuenta que este autor nunca se ha esforzado por escribir en neerlandés como un autor nativo. Todo lo contrario. Parte del encanto de su obra radica en el hecho de que su neerlandés causa extrañeza en los hablantes nativos.

Kader Abdolah, seudónimo de Hossein Sadjadi Ghaemmaghami Farahani, es un escritor iraní que lleva viviendo desde 1988 en los Países Bajos, donde se le concedió la condición de refugiado político. Nacido en 1954 en Arak, estudió Física en la Universidad de Teherán. Acabó involucrándose en los movimientos de resistencia,

1. https://letterenfonds.secure.force.com/vertalingendatabase/zoeken?type=search.

Philippe HUMBLÉ Tres traducciones españolas de Kader Abdolah 
primero contra el Sah y después contra el régimen islámico. Una vez en los Países Bajos, comenzó a escribir literatura en neerlandés. En 1993 publicó su primer libro, De Adelaars ("Las Águilas»), que ganó el premio al mejor debut en lengua neerlandesa. De su novela Het huis van de moskee (La casa de la mezquita), publicada en 2005, se vendieron más de 100000 ejemplares, una cifra muy elevada teniendo en cuenta que el neerlandés sólo tiene 25 millones de hablantes. La obra fue traducida a una docena de idiomas, entre ellos el español.

Este artículo se centra en la traducción de las tres novelas de Kader Abdolah que han sido traducidas al español hasta la fecha: De reis van de lege flessen (El viaje de las botellas vacías), Spijkerschrift (El reflejo de las palabras) y Het huis van de moskee (La casa de la mezquita).

Los críticos holandeses (no hemos encontrado reseñas flamencas) no siempre ven con buenos ojos el peculiar uso que Kader Abdolah hace de la lengua. Algunos han censurado su simplicidad exagerada, su manera casi ingenua de escribir. Peppelenbos (2011) es uno de ellos: «Cuando Abdolah escribe dos o tres frases seguidas, pasa a la línea siguiente. Es una manera algo infantil de escribir»². Otras muchas personas, sin embargo, aprecian justamente ese minimalismo. El hecho de que, en la Semana del Libro de 2007, Het huis van de moskee fuera elegido por el público como segundo mejor libro en neerlandés de todos los tiempos, solo por detrás de De ontdekking van de wereld (El descubrimiento del cielo) de Harry Mulisch, es prueba suficiente de ello.

No cabe duda de que el autor tiene un mensaje que transmitir, y es de suponer que el estilo forma parte de ese mensaje. La peculiar manera de escribir de Kader Abdolah es, sin embargo, lo que más peligro corre al pasar por una traducción. El estilo es algo que se pierde fácilmente. Por otro lado, si las novelas de Kader Abdolah tienen éxito en otras lenguas como el español, ¿es porque su mensaje ha sido trasmitido correctamente, con independencia del vehículo lingüístico, o porque los traductores han sabido plasmar también su particular estilo? En lo que sigue, analizaremos sobre todo la relación type/token, que nos puede informar sobre la densidad léxica de un texto.

\section{LA RELACIÓN TYPE/TOKEN}

Investigaremos las traducciones de Kader Abdolah con la ayuda de un concepto procedente de la lingüística de corpus, que es la relación type/token. Asimismo, compararemos el número de oraciones de cada texto para ver si este dato corrobora las conclusiones que sacamos del análisis de la relación type/token. Esta compara el

2. «Als Abdolah twee, drie zinnen achter elkaar heeft geschreven, begint hij alweer op de volgende regel. Een kinderlijke manier van schrijven».

Philippe HUMBLÉ Tres traducciones españolas de Kader Abdolah 
número de ocurrencias únicas de una palabra con el número total de palabras en un texto. Si este presenta una gran variedad de formas diferentes, la relación type/token será más elevada, porque habrá más palabras que aparecen solamente una vez.

La relación type/token puede facilitarnos información valiosa si la interpretamos con precaución. El principal argumento contra su uso es que el tamaño del texto influye directamente en los resultados. Como en nuestro caso se trata de traducciones, los textos comparados tienen un tamaño similar.

Hay otras críticas que se pueden hacer a este instrumento, sobre todo cuando se comparan textos en lenguas diferentes. En el caso del par neerlandés-español, encontramos diferencias morfológicas y ortográficas que influyen tanto en el número de types como en el número de tokens. Con respecto a los verbos, por ejemplo, los dos idiomas difieren en el número de tipos verbales disponibles y cuáles de ellos son de uso obligatorio. En neerlandés, un verbo regular tiene seis formas diferentes posibles, mientras que en español contamos hasta 48. Simplificando, podríamos decir que, por término medio, cada verbo neerlandés puede ser traducido al castellano por ocho formas diferentes. Una traducción del neerlandés al español tendría, solo por esta razón, más types. Una segunda advertencia a tener en cuenta es el tratamiento diferente que se da en neerlandés a palabras compuestas de varios elementos (multiword units). En neerlandés, los diferentes elementos se escriben en una sola palabra (voedselbedeling), mientras que en español se separan (distribución de alimentos), lo que aumenta el número de tokens.

Por estas razones, la comparación de relaciones type/token de un texto se tiene que manejar con cierto cuidado, pero ello no impide que el procedimiento dé una idea de la densidad de un texto original comparado con la densidad de su traducción.

\section{LOS UNIVERSALES DE LA TRADUCCIÓN}

Toda esta problemática guarda estrecha relación con el debate sobre los «universales de la traducción». Cuando a principios de los años noventa, el ordenador pasó a convertirse en una herramienta común en la investigación, los lingüistas rápidamente quisieron utilizar las nuevas tecnologías para obtener un conocimiento más profundo del comportamiento de la lengua por medio de la exploración de grandes cantidades de texto. Los teóricos de la traducción pronto le auguraron un gran futuro a la rama de la lingüística aplicada. Baker lo formulaba de la manera siguiente en 1993:

[...] we are about to witness a turning point in the history of the discipline. I would like to argue that this turning point will come as a direct consequence of access to large corpora of both original and translated texts, and of the development of specific methods and tools for interrogating such corpora in ways which are appropriate to the needs of translation scholars. (Baker 1993, 235)

Philippe HUMBLÉ Tres traducciones españolas de Kader Abdolah 
Pocos años antes, los estudios descriptivos de traducción habían tomado impulso con Lambert (1978), Even-Zohar (1979) y Toury (1980). Las «normas» eran muy importantes desde esa perspectiva e implicaban la búsqueda de "universales». En el artículo arriba citado, Baker establece una lista de todos los elementos que sospechaba que entrarían a formar parte de las futuras hipótesis de investigación en busca de «universales»:

(i) A marked rise in the level of explicitness compared to specific source texts and to original texts in general [...]

(ii) A tendency towards disambiguation and simplification. [...]

(iii) A strong preference for conventional "grammaticality". [...]

(iv) A tendency to avoid repetitions which occur in source texts, either by omitting them or rewording them [...]

(v) A general tendency to exaggerate features of the target language. [...]

(vi) Point (v) above notwithstanding, it has been shown that the process of mediation often results in a specific type of distribution of certain features in translated texts vis-àvis source texts and original texts in the target language. (Baker 1993, 243-245)

En un principio, estas hipótesis sonaban correctas y casi parecían de sentido común. Laviosa, por su parte, abordaría en un artículo de 1998 la cuestión de los «universales» específicamente en la traducción al inglés. Concluía así:

In summary, we can say that four main global patterns appear to characterize newspaper and narrative translational texts in English:

i) Translated texts have a relatively lower percentage of content words versus grammatical words (i.e. their lexical density is lower);

ii) The proportion of high frequency words versus low frequency words is relatively higher in translated texts;

iii) The list head of a corpus of translated texts accounts for a larger area of the corpus (i.e. the most frequent words are repeated more often);

iv) The list head of translated texts contains fewer lemmas.

I propose to call these regular features of translated text "core patterns of lexical use» in an attempt to convey the fact that because they occur in two different subject domains, they may prove typical of English translated text in general. (Laviosa 1998, 8)

Laviosa menciona particularmente el concepto de "densidad léxica», que es el concepto que nos interesa más específicamente aquí. Xiao (2010, 7-12) ofrece un resumen sistematizado de todas las características presentadas como candidatas a universales de la traducción en las últimas décadas. La explicitación, la simplificación y la normalización han sido los fenómenos más investigados, pero en ninguno de ellos se ha alcanzado un consenso. La simplificación parece tener muchas posibilidades

Philippe HUMBLÉ Tres traducciones españolas de Kader Abdolah 
de éxito, aunque incluso a este respecto surgen voces discordantes. Para el ámbito médico y técnico, por ejemplo, Corpas Pastor llegó a la siguiente conclusión:

To sum up, the results of our experiments suggest that simplification does affect translated texts, albeit this is not true with regard to the sentence length and the use of simple vs. complex sentences, and texts produced by non-professional translators do not seem to possess such simplification traits. (Corpas Pastor et al. 2008, 81)

Sin embargo, en 2010, en otro estudio basado en textos médicos y técnicos (llisei et al. 2010, 510), en el que se aplicaron herramientas computacionales más sofisticadas, quedó de manifiesto que las traducciones son identificables y que los resultados obtenidos pueden considerarse como un argumento para la existencia del universal de la simplificación. Parece, en efecto, natural que un traductor tenga tendencia a simplificar y no a "complicar» el original.

El caso de la retraducción literaria parece confirmar esta hipótesis. Dependiendo de su éxito y de su recepción por parte de la crítica, una obra literaria se traduce dos veces o más, en distintos momentos, y por diferentes traductores. Algunos críticos, entre ellos nada menos que Berman (1990, citado por Dastjerdi 2013, 180), han propuesto la hipótesis de que una primera traducción estaría necesariamente más orientada a facilitar el acceso a la obra traducida por parte del público: «[...] the first translation serves as an introduction of the foreign work to the target readers, hence the need for new source-oriented retranslation in which the focus is on the letter and style of the source text».

A continuación se analizan los resultados del estudio de la relación type/token para las tres novelas de Kader Abdolah que han sido traducidas al español hasta la fecha, comparando la traducción con el texto original.

\section{LA RELACIÓN TYPE/TOKEN EN TRES NOVELAS DE KADER ABDOLAH Y SUS TRADUCCIONES}

\subsection{De reis van de lege flessen (1997) - El viaje de las botellas vacías (1999)}

El viaje de las botellas vacías fue uno de los primeros libros de Kader Abdolah y el primero en tener cierto éxito. Publicado en 1997, cuenta la historia de Bolfazl con toda probabilidad el alter ego de Kader Abdolah - durante sus primeros meses en los Países Bajos. Es una novela corta que retrata de manera vívida, cómica y a veces un poco triste, la adaptación de un exiliado a un país del que nunca habría pensado que se fuera a convertir en su segunda patria. El libro ha sido traducido 
a varias lenguas, entre ellas el alemán, el bosnio, el francés, el noruego, el turco y el español.

Como todas las obras de Kader Abdolah, El viaje de las botellas vacías se distingue por un estilo sencillo y un vocabulario más bien limitado que delatan la «extranjeridad» de su autor. Es más, parece que el autor hace todo lo posible por recalcar su origen persa. Cuando comparamos el original con su traducción por medio de datos objetivos como los proporcionados por el análisis computacional, llegamos a los siguientes resultados:

\begin{tabular}{|l|l|l|l|}
\hline & Types & Tokens & Standardized Type/token \\
\hline De reis van de lege flessen & 4149 & 39518 & 38,46 \\
\hline El viaje de las botellas vacías & 4426 & 35118 & 41,59 \\
\hline
\end{tabular}

Tabla 1. Type/token en De reis van de lege flessen

En este caso, se nota que la relación type/token es más alta en la traducción que en el original. Si bien la diferencia no es muy grande, resulta significativa, ya que se espera una densidad léxica menor en la traducción. Al parecer, la traducción contiene más palabras diferentes que el original. Como veremos a continuación, ocurre algo similar en el caso de Spijkerschrift.

\subsection{Spijkerschrift (2000) - El reflejo de las palabras (2006)}

La novela Spijkerschrift (2000), literalmente «escritura cuneiforme», fue publicada en español como El reflejo de las palabras (2006). Cuenta la historia de un escritor iraní exiliado en los Países Bajos que un día recibe por correo un cuaderno escrito por su por entonces ya difunto padre, analfabeto, sordo y mudo, como lo era el padre de Kader Abdolah. La escritura es apenas legible, lo que obliga al autor a reconstruir la mayor parte de la historia de su progenitor, confiando en sus recuerdos. El cuaderno ni siquiera está escrito en caracteres normales. El narrador califica la escritura de su padre de "cuneiforme», como las inscripciones en una cueva cerca de su pueblo natal que su padre frecuentaba. Así, el libro cuenta la propia historia del autor, la historia de su familia y también la de Irán en el siglo XX. Resulta ser un homenaje del autor a su padre discapacitado, en el que describe una relación en la que un niño tiene que desempeñar simultáneamente el papel de padre, hermano y amigo de su progenitor. El mensaje que se transmite a través de la obra original en neerlandés es la historia de un autor que escribe desde un punto de vista no nativo. El original en lengua neerlandesa se presenta como la traducción de un escrito iraní casi ilegible y, de hecho, suena 
como una traducción. Es una traducción en la que el traductor elige no ser invisible. El original es, por tanto, una traducción «extranjerizante» desde el principio (Venuti 1995). Esto se refleja en el vocabulario algo limitado y en el uso de frases breves y abruptas, generalmente respetando la secuencia sujeto-verbo-objeto. Es como si el escritor dijera: «Soy un exiliado, un extranjero que no escribe perfectamente, pero que tiene éxito contando historias de una manera diferente».

En El reflejo de las palabras, la diferencia de densidad léxica entre el original y su traducción es incluso mayor.

\begin{tabular}{|l|l|l|l|}
\hline & Types & Tokens & Standardized Type/token \\
\hline Spijkerschrift & 6746 & 84604 & 39,77 \\
\hline El reflejo de las palabras & 9997 & 81998 & 46,24 \\
\hline
\end{tabular}

Tabla 2. Type/token en Spijkerschrift

Aquí constatamos que la diferencia entre original y traducción es de más de seis puntos, por lo que podemos concluir que el traductor ha enriquecido notablemente el lenguaje de Kader Abdolah.

\subsection{Het huis van de moskee (2005) - La casa de la mezquita (2008)}

La casa de la mezquita (2005) es la novela de más éxito de Kader Abdolah hasta la fecha. El libro describe la vida de una familia durante el régimen del último Sah y la revolución de Jomeini de 1979. Termina con la muerte de este. La historia transcurre en una ciudad ficticia, pero tiene un fuerte sabor real. Tal y como se ha comentado antes, en la Semana del Libro de 2007 fue el segundo libro más votado en el ranking de libros más populares en lengua neerlandesa de todos los tiempos. Ha sido traducido al búlgaro, checo, chino, escocés, eslovaco, español, finés, griego, húngaro, serbio, sueco e incluso al farsi.

\begin{tabular}{|l|l|l|l|}
\hline & Types & Tokens & Standardized Type/token \\
\hline Het huis van de moskee & 8887 & 117476 & 40,99 \\
\hline La casa de la mezquita & 11455 & 112772 & 44,97 \\
\hline
\end{tabular}

Tabla 3. Type/token en Het Huis van de moskee 
En el caso de Het Huis van de moskee, la diferencia de densidad léxica entre el original y la traducción también es marcada. La densidad léxica de la traducción supera en cuatro puntos la del original.

\subsection{Comparación con un corpus de novelas en español y en neerlandés}

Podemos comparar estos resultados con los de una novela común, tanto en neerlandés como en español.

\begin{tabular}{|l|l|}
\hline & Standardized Type/Token \\
\hline Vila Matas - Doctor Pasavento & 44,67 \\
\hline Javier Marías - Corazón tan Blanco & 44,96 \\
\hline Lezama Lima - Paradiso & 47,27 \\
\hline Belli - La mujer habitada & 48,11 \\
\hline Laforet - Nada & 46,78 \\
\hline Delibes - La Sombra del Ciprés & 49,46 \\
\hline
\end{tabular}

Tabla 4. Relación type/token en seis novelas contemporáneas en español

Como podemos constatar, la relación media type/token de una novela común en español oscila entre 44 y 49. Las traducciones de Kader Abdolah, por lo tanto, tienden a acercarse a esta «norma» en castellano. Las novelas en neerlandés arrojan resultados parecidos.

\begin{tabular}{|l|l|}
\hline & Standardized Type Token \\
\hline Herman Brusselmans - Gugenheimer wast witter & 42,40 \\
\hline Jeroen Brouwers - Bezonken rood & 43,78 \\
\hline Hugo Claus - Het verdriet van België & 47 \\
\hline Peter Bruwalda - Bonita Avenue & 49,17 \\
\hline
\end{tabular}

Tabla 5. Relación type/token de seis novelas contemporáneas en neerlandés

En este caso, el valor oscila entre 42 y 49, lo que indica que ambas lenguas comparten valores comunes situados entre 42 y 50 . Estos datos demuestran que los 
valores de los originales de Kader Abdolah son excepcionalmente bajos, lo cual nos permite confirmar que los traductores han «enriquecido» el texto.

\section{NÚMERO DE ORACIONES EN TRES NOVELAS DE KADER ABDOLAH Y SUS TRADUCCIONES}

Otra manera de estudiar los cambios introducidos por los traductores consiste en contar el número de oraciones. Este número indica en qué medida los traductores añaden conectores, lo que apuntaría a una «sofisticación» del texto. Contando el número de puntos, puntos de interrogación y puntos de exclamación, llegamos a los siguientes resultados.

\begin{tabular}{|l|l|l|l|l|l|l|}
\hline & $\begin{array}{l}\text { De reis van } \\
\text { de lege } \\
\text { flessen }\end{array}$ & $\begin{array}{l}\text { El viaje de } \\
\text { las botellas } \\
\text { vacías }\end{array}$ & Spijkerschrift & $\begin{array}{l}\text { El reflejo } \\
\text { de las } \\
\text { palabras }\end{array}$ & $\begin{array}{l}\text { Het Huis } \\
\text { van de } \\
\text { moskee }\end{array}$ & $\begin{array}{l}\text { La casa } \\
\text { de la } \\
\text { mezquita }\end{array}$ \\
\hline Punto & 4064 & 4134 & 7336 & 6206 & 7278 & 7162 \\
\hline $\begin{array}{l}\text { Punto de } \\
\text { interrogación }\end{array}$ & 587 & 574 & 947 & 981 & 748 & 751 \\
\hline $\begin{array}{l}\text { Punto de } \\
\text { exclamación }\end{array}$ & 146 & 152 & 328 & 459 & 744 & 660 \\
\hline $\begin{array}{l}\text { Total de } \\
\text { oraciones }\end{array}$ & 4797 & 4860 & 8611 & 7646 & 8770 & 8573 \\
\hline & & & & & & \\
\hline & $+1,35 \%$ & & -965 & & -197 \\
\hline
\end{tabular}

Tabla 6. Número de oraciones en tres novelas de Kader Abdolah

El resultado de este cómputo está en correlación con la relación type/token. Es evidente que el traductor de Spijkerschrift es el que más oraciones ha unido para convertirlas en oraciones coordinadas o subordinadas. Se trata de un $11,2 \%$ de todas las oraciones, lo cual es mucho. Es también el traductor que más «ha enriquecido» el texto original desde el punto de vista léxico.

Donde menos se ha intervenido en el número de oraciones es en la traducción de De reis van de lege flessen, que presenta también la menor densidad léxica. Dicho de otro modo, las dos tendencias se confirman y muestran que ha habido, sobre todo en el caso del traductor de Spijkerschrift, un intento de sofisticación del original, no 
solo a nivel de vocabulario, sino también a nivel de coordinación/subordinación de las oraciones.

\section{CONCLUSIONES}

La primera conclusión es que, a diferencia de lo que sugiere la teoría de los universales de la traducción, en todas las traducciones al español de Kader Abdolah los traductores aumentan la densidad léxica en lugar de reducirla. Esta tendencia es muy pronunciada en el caso de El reflejo de las palabras, mientras que pierde algo de fuerza en La casa de la mezquita y sobre todo en El viaje de las botellas vacías, aunque sigue siendo significativa. En otro estudio anterior sobre la traducción de Spijkerschrift al francés y al inglés registramos la misma tendencia.

\begin{tabular}{|l|l|}
\hline & $\begin{array}{l}\text { Relación type/token } \\
\text { Standardized }\end{array}$ \\
\hline Spijkerschrift & 39,69 \\
\hline My Father's Notebook & 39,34 \\
\hline Cunéiforme & 41,60 \\
\hline El reflejo de las palabras & 46,24 \\
\hline & \\
\hline Het huis van de moskee & 40,99 \\
\hline The house of the mosque & 42,72 \\
\hline La casa de la mezquita & 44,97 \\
\hline
\end{tabular}

Tabla 7. Relación type/token en cinco novelas traducidas de Kader Abdolah

Lo cierto es que el peculiar estilo de Kader Abdolah, tan evidente para un lector neerlandófono, se pierde en todas la traducciones. La buena noticia es que la fama del autor no depende, por tanto, de lo que Borges (1976) hubiera llamado «la supersticiosa ética del lector». Es decir, Kader Abdolah sigue siendo un autor de éxito, aun cuando se pierdan los rasgos típicos de su estilo extranjerizante.

Ahora bien, si la teoría de los universales de la traducción se confirma en la mayoría de casos, ¿por qué no es así en el caso de Kader Abdolah? La impresión es que los traductores tienden a «normalizar» el texto no solamente en el sentido de la gramática, sino también en el sentido del léxico. Se podría pensar que, inconscientemente, los traductores han hecho lo posible para que las novelas traducidas de Kader Abdolah se 
parezcan a la novela media de un escritor hispanohablante nativo. Y, aparentemente, los traductores de las otras lenguas han hecho lo mismo. Todos han elevado el nivel léxico, en detrimento del «acento inmigrante» del original.

Podemos preguntarnos si todo esto tiene que ver con el hecho de que el neerlandés es una «lengua minoritaria». En otras palabras, ¿tendrá un traductor de una lengua «mayoritaria» - o sea una lengua de la que se traduce con frecuencia- tendencia a traducir una obra de una lengua minoritaria con menos cautela? ¿La adaptará con mayor facilidad a lo que él perciba como «normalidad»? Sería comprensible desde un punto de vista psicológico, aunque difícil de averiguar científicamente. Uno podría pensar que un traductor que traduce del inglés puede llegar a sentirse más vigilado que un traductor que traduce del neerlandés.

Hay otra incógnita, que es la personalidad de los traductores. La información contenida en la base de datos del Nederlands Letterenfonds y el Vlaams Fonds voor de Letteren nos permite afirmar que los tres traductores tenían probada experiencia en el ámbito de la traducción literaria en el momento de afrontar la traducción de la novela de Kader Abdolah, de modo que los resultados de nuestro estudio no pueden ser atribuidos a una falta de conocimiento o destreza. Resultan especialmente ilustrativas las palabras que Diego Puls dedicó a su traducción de Spijkerschrift en la entrevista que le hicieron con motivo de la entrega del Premio Letterenfonds 2010: «Es un libro difícil de traducir. Por el estilo. Abdolah escribe como un niño de 14 años. Su neerlandés es bastante simple. A los lectores holandeses eso les gusta, pero a un lector de habla española le resulta demasiado torpe. Es algo que he tenido que arreglar un poco en la traducción»³. Según decía, había quedado sorprendido por el éxito del que gozaba Kader Abdolah en Argentina, donde el libro iba ya por la segunda edición, algo que aún no le había comunicado el editor.

La teoría de los universales de la traducción siempre ha sido controvertida, porque busca demostrar que la balanza tiene que inclinarse necesariamente en una u otra dirección. Sin embargo, puede ser que el translationese, como se le ha llamado con cierto desdén, no sea ni una tendencia a la simplificación ni a la sofisticación, sino una preferencia por cumplir con las «normas» del público lector meta.

3. «lk vond het een lastig boek om te vertalen, vanwege de stijl. Abdolah schrijft als een jongen van 14 jaar. Een heel simpel soort Nederlands. Nederlandse lezers vinden dat leuk, maar voor een Spaanstalige lezer is het te onbeholpen. Ik heb dat in de vertaling een beetje moeten opschonen».

Philippe HUMBLÉ Tres traducciones españolas de Kader Abdolah 


\section{REFERENCIAS BIBLIOGRÁFICAS}

BAKER, Mona. 1993. "Corpus Linguistics and Translation Studies - Implications and Applications». En Text and Technology: In honour of John Sinclair, ed. por Mona Baker, Gill Francis y Elena Tognini-Bonelli. Amsterdam/Philadelphia: John Benjamins, 233-250.

BÜCHLER, Alexandra y Giulia TRENTACOSTI. 2015. Publishing Translated Literature, in the United Kingdom and Ireland 1990-2012 (statistical report). Aberystwyth University, Wales: Mercator Institute for Media, Languages and Culture.

BORGES, Jorge Luis. 1976. Discusión. Madrid: Alianza/Emecé.

BRANCHADELL, Albert y Lovell Margaret WEST. 2004. Less Translated Languages. Amsterdam: John Benjamins (Benjamins Translation Library, 58).

Casanova, Pascale. 2015. La Langue Mondiale: Traduction et Domination. Paris: Seuil.

CORPAS PAStOR, Gloria, Mitkov RuSLAN, Afzal NAVEEd y Viktor PEKAR. 2008. «Translation Universals: Do They Exist? A Corpus-Based NLP Study of Convergence and Simplification". En Proceedings of the 8th Conference of the Association for Machine Translation in the Americas (AMTA-2008). Hawaii, 21-25 October 2008, 77-81.

DASTJERDI, Hossein Vahid y Amene MOHAMMADI. 2013. «Revisiting 'Retranslation Hypothesis': A Comparative Analysis of Stylistic Features in the Persian Retranslations of Pride and Prejudice». Open Journal of Modern Linguistics 3: 174-81.

Even-ZOHAR, Itamar. 1979. «Polysystem Theory». Poetics Today 1 (1-2, Autumn): 287-310.

-. 2010. Papers in Culture Research. Tel Aviv: Unit of Culture Research, Tel Aviv University.

HUMBLÉ, Philippe. 2016. «Translating Exile: Two Translations of Kader Abdolah's Spijkerschrift in the Light of Corpus Analysis». En Input a Word, Analyse the World. Selected Approaches to Corpus Linguistics, ed. por Francisco Alonso Almeida. Newcastle upon Tyne: Cambridge Scholars Publishing, 399-414.

ILISEI, Iustina, Diana INKPEN, Gloria CORPAS PASTOR y Ruslan MITKOV. 2010. «ldentification of Translationese: A Machine Learning Approach». En Computational Linguistics and Intelligent Text Processing. Berlin/Heidelberg: Springer, 503-511.

LAMBERT, Jose, James S. HOLMES y Raymond VAN DEN BROECK. 1978. Literature and Translation: New Perspectives in Literary Studies with a Basic Bibliography of Books on Translation Studies. Leuven: Acco.

LAVIOSA, Sara. 1998. "Core Patterns of Lexical Use in a Comparable Corpus of English Narrative Prose'». Meta: Journal des traducteurs 43 (4): 557-70.

LINN, Stella. 2006. "Trends in the Translation of a Minority Language: The Case of Dutch". En Sociocultural Aspects of Translating and Interpreting, ed. por Anthony Pym, Miriam Shlesinger y Zuzana Jettmarova. Amsterdam /Philadelphia: John Benjamins Publishing (Benjamins Translation Library, 67), 27-39.

MALMKJAER, Kirsten y Daniel Gile. 2001. Claims, Changes and Challenges in Translation Studies Selected Contributions from the EST Congress. København: Gyde Hansen Copenhagen Business School.

PePPelenBOS, Coen. 2011. "Recensie van De kraai». Leeuwarder Courant (11 de marzo de 2011).

TOURY, Gideon. 1980. Translation Theory: a Reader. Tel Aviv University: Dyonun.

Philippe HUMBLÉ Tres traducciones españolas de Kader Abdolah 
NEDERLANDS Letterenfonds. 2010. Nederlands Letterenfonds Prijzen 2010 voor de vertaler als cultureel bemiddelaar: Mariolein Sabarte Belacortu, Hans Driessen, Diego Puls.

XIAO, Richard. 2010. "How Different Is Translated Chinese from Native Chinese?: A CorpusBased Study of Translation Universals». International Journal of Corpus Linguistics 15 (1): $5-35$.

\section{Novelas en neerlandés}

ABDOLAH, Kader. 2011 [1. ${ }^{a}$ ed. 1997]. Spijkerschrift. Breda: De Geus.

-. 2012 [1. ${ }^{a}$ ed. 2000]. De reis van de lege flessen. Breda: De Geus.

-. 2014 [1. ${ }^{a}$ ed. 2005]. Het huis van de moskee. Breda: Colibri.

\section{Traducciones}

ABDOLAH, Kader. 1999. El viaje de las botellas vacías. Traducido por Andrea Morales Vidal. Barcelona: Galaxia Gutenberg Círculo de Lectores.

-. 2003. Cunéiforme. Traducido por Isabelle Rosselin. Paris: Gallimard.

-. 2006. My Father's Notebook. Traducido por Susan Massotty. Edinburgh/New York: Canongate Books.

-. 2006. El reflejo de las palabras. Traducido por Diego Puls Kuipers. Barcelona: Salamandra.

-. $\quad$ 2008. La casa de la mezquita. Traducido por Marta Arguilé. Barcelona: Salamandra.

-. 2010. The house of the mosque. Traducido por Susan Massotty. Edinburgh/New York: Canongate. 